\title{
催化不对称[8+2]环加成反应构建环庚三烯并吡咯烷-3,3'-吲哚酮
}

\author{
谢明胜武晓霞王刚林丽丽冯小明* \\ (四川大学 绿色化学与技术教育部重点实验室 化学学院 成都 610064)
}

\begin{abstract}
摘要 设计了 azaheptafulvenes 作为偶极子与 $N$-Boc-3-烯基吲哚酮反应, 用来构建螺环 [吡咯烷-3,3'-吲哚酮]衍生物. 在 1 $\mathrm{mol} \%$ 手性氮氧-Ni(II)配合物催化剂条件下，该不对称 $[8+2]$ 环加成反应以高的收率( $90 \%$ \% $\%$ 收率), 好的非对映选择 性(高达 $97: 3 d r$ )和优异的对映选择性(92\% 99\% ee)得到手性环庚三烯并螺环[吡咯烷-3,3'-弜哚酮]衍生物.
\end{abstract}

关键词 手性氮氧; 镍; [8+2]环加成; 螺环; 不对称催化

\section{Catalytic Asymmetric [8+2] Cycloaddition for the Construction of Cycloheptatriene-Fused Pyrrolidin-3,3'-Oxindoles}

\author{
Xie, Mingsheng Wu, Xiaoxia Wang, Gang Lin, Lili Feng, Xiaoming* \\ (Key Laboratory of Green Chemistry \& Technology, Ministry of Education, College of Chemistry,
} Sichuan University, Chengdu 610064)

\begin{abstract}
The spiro pyrrolidin-3,3'-oxindole core is a privileged skeleton that is found in many natural products and biologically active molecules. For its construction, the [3+2] cycloaddition of $2 \pi$ component 3 -alkenyl-oxindoles with $3 \pi$ component azomethine ylides is one of the most effective strategies. Since the pioneering work of Gong and co-workers, great effort has been devoted to the development of asymmetric [3+2] cycloadditions with azomethine ylides as the dipole synthon, and impressive progress has been achieved. In contrast, there is no report about higher-order [8+2] cycloaddition of 3-alkenyl-oxindoles with azaheptafulvenes as the $8 \pi$ dipole synthons, which is an effective way to build cycloheptatriene-fused pyrrolidin-3,3'-oxindole derivatives with three contiguous stereocenters, including a spiro-quaternary chiral carbon atom. Herein, the azaheptafulvenes is developed as a new dipole synthon with 3-alkenyl-oxindoles to construct pyrrolidin-3,3'-oxindoles derivatives for the first time. In the presence of $1 \mathrm{~mol} \%$ of chiral $N, N^{\prime}$-dioxide $\mathbf{L 6}$-Ni(II) complex, the asymmetric [8+2] cycloaddition performs well, affording functionalized cycloheptriene-fused pyrrolidin-3,3'-oxindoles derivatives in excellent yields $(90 \% \sim 99 \%)$, diastereoselectivities (up to $97: 3 d r$ ), and enantioselectivities $(92 \% \sim 99 \%$ ee) under mild conditions. A representative procedure for the asymmetric [8+2] cycloaddition is as follows: to a test tube, the catalyst solution ( $1 \mathrm{~mol} \%$, in THF) was added, and the THF was evaporated by oil pump. $N$-Boc-3-alkenyl-oxindole $2 \mathrm{a}$ and $0.5 \mathrm{~mL}$ of $\mathrm{CH}_{2} \mathrm{Cl}_{2}$ were added sequentially under air $\left(\mathrm{N}_{2}\right.$ atmosphere is not necessary). The reaction solution was stirred at $35{ }^{\circ} \mathrm{C}$ for $0.5 \mathrm{~h}$, then the $8 \pi$ component azaheptafulvene 1a (1.05 equiv.) was added. The mixture continued stirring at $35{ }^{\circ} \mathrm{C}$ until $\mathrm{N}$-Boc-3-alkenyl-oxindole 2a was consumed (detected by TLC). Finally, the corresponding product 3a was purified by flash chromatography on basic $\mathrm{Al}_{2} \mathrm{O}_{3}[V$ (petroleum $\left.) / V\left(\mathrm{CH}_{2} \mathrm{Cl}_{2}\right) / V(\mathrm{EtOAc})=8: 8: 1\right]$.

Keywords chiral $N, N^{\prime}$-dioxide; $\mathrm{Ni}(\mathrm{II}) ;[8+2]$ cycloaddition; spiro; asymmetric catalysis
\end{abstract}

\section{1 引言}

螺环一吡咯烷-3,3'-吲哚酩骨架存在于许多天然产物 和具有生物活性的化合物中(图 1), 如 spirotryprostain A 和 $\mathrm{B}$, isocorynoxeine 等 ${ }^{[1,2]}$, 其合成受到化学工作者广泛 关注. 如何通过合成化学方法高效高选择性的构筑螺 环一吡咯烷-3,3'-吲哚酮手性骨架一直是化学工作者努力 的方向. 在众多构建螺环一吡咯烷-3,3'-吲哚酮的方法中, 3 -烯基吲哚酮作为 $2 \pi$ 电子受体与甘氨酸酯亚胺作为 $3 \pi$ 电子供体的 $[3+2]$ 环加成反应是最有效的合成方法之一
(图 2) ${ }^{[2 \sim 5]}$.

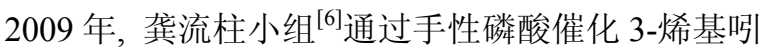
哚酮、醛和氨基酸酯的三组分不对称 $[3+2]$ 环加成反应, 实现了首例催化不对称合成螺环一吡咯烷-3,3'-吲哚酮. 此后, 通过 3-烯基吲哚酤与甘氨酸酯亚胺的不对称 $[3+$ 2]环加成反应来构建该类化合物的方法被陆续报道, 在 催化体系和底物范围方面都取得了极大的进展 ${ }^{[7,8]}$. 但 目前尚没有通过高阶环加成反应来构建螺环一吡咯烷3,3'-吲哚酮衍生物的报道 ${ }^{[9 \sim 17]}$. 2013 年, 我们利用手性

*E-mail: xmfeng@scu.edu.cn; Fax: +86-028-85418249; Tel: +86-028-85418249

Received December 14, 2013; published January 9, 2014.

Supporting information for this article is available free of charge via the Internet at http://sioc-journal.cn.

Project supported by the National Basic Research Program of China (973 Program: No. 2011CB808600), the National Natural Science Foundation of China (Nos. 21321061, 21290182 and 21172151) and Ministry of Education (No. 20110181130014).

项目受国家重点基础研究发展计划(973 计划：No. 2011CB808600)、国家自然科学基金(Nos. 21321061, 21290182 和 21172151 )和教育部(No. 20110181130014)资助. 
<smiles>COc1ccc2c(c1)NC(=O)[C@]21C[C@H]2C(=O)N3CCC[C@H]3C(=O)N2C1C=C(C)CO[Mg]</smiles><smiles>CC(C)=CC1N2C(=O)C3CCCN3C(=O)C2=CC12C(=O)Nc1ccccc12</smiles>

Spirotryprostatin B

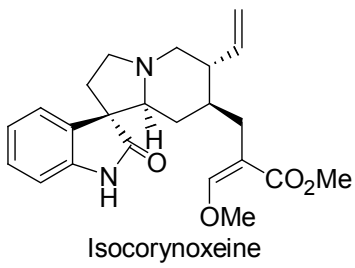

图 1 代表性含手性螺环-吡咯烷-3,3'-吲哚酮骨架的天然产物

Figure 1 Selected bioactive natural products bearing the ring-fused pyrrolidin-3,3'-oxindole skeleton

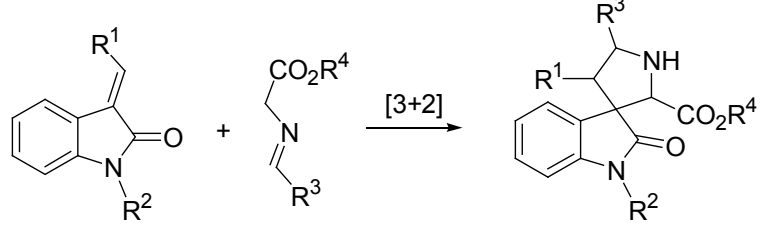

图 $2[3+2]$ 环加成反应构建螺环一吡咯烷-3,3'-吲哚酮类化合物

Figure $2[3+2]$ cycloaddition for the synthesis of spiro pyrrolidin-3,3'-oxindole derivatives

双氮氧酰胺-镍(II)配合物为催化剂, 第一次实现了催化 不对称 $[8+2]$ 环加成反应 ${ }^{[18]}$. 反应以 azaheptafulvenes 作 为 $8 \pi$ 电子供体, 烯酯作为电子受体, 可高对映选择性地 合成一系列环庚三烯并吡咯类化合物. 在后续研究中, 我们希望以 azaheptafulvenes 作为 $8 \pi$ 电子供体, 3 -烯基吲 哚酮作为 $2 \pi$ 电子受体, 通过催化不对称 $[8+2]$ 环加成反 应来高效、高立体选择性地构建螺环-吡咯烷-3,3'-吲哚 酮类化合物. 这类产物具有三个连续的手性中心, 包括 一个螺环-季碳手性中心, 合成具有一定挑战性.

通常认为 $[8+2]$ 环加成反应是分步历程进行的(图 3): 首先, azaheptafulvenes 中的氮原子利用其亲核性, 对 3-烯基吲哚醖进行亲核加成反应, 形成两性离子中间 体. 其中, $8 \pi$ 电子组分显电正性, 其电子以离域的形式 分布在七元环上; 吲哚酮组分中羰基的 $\alpha$ 位则具电负性. 环合生成吡咯烷结构, 得到相应的环庚三烯并吡咯烷3,3'-吲哚酮类化合物 ${ }^{[19-21]}$. 其中环庚三烯的三个共轭双 键极易互变, 发生烯丙基迁移和 [1,5]-氢迁移等异构化 反应, 生成其它不易分离的异构体 ${ }^{[22]}$. 由于这些异构体 在柱层析中具有近乎相同的 $R_{\mathrm{f}}$ 值, 难以对其分离纯化, 因而如何抑制产物的异构化也是该 $[8+2]$ 环加成反应需 要解决的问题之一.

近年来, 我们课题组设计和发展了一类手性双氮氧 酰胺一金属配合物催化剂, 在一系列不对称催化反应中<smiles>[R]C=c1c(=[OH+])n([R])c2ccccc2/c1=C/[R]</smiles>

图 $3[8+2]$ 环加成反应构建环庚三烯并吡咯烷-3,3'-吲哚酮类化合物 Figure $3[8+2]$ cycloaddition for the synthesis of cycloheptatrienefused pyrrolidin-3,3'-oxindoles derivatives

显示出了优异的催化效果 ${ }^{[23]}$. 我们继续将这类手性双 氮氧一金属配合物用于催化 azaheptafulvenes 与 3-烯基吲 哚酮的不对称 $[8+2]$ 环加成反应，构建系列手性环庚三 烯并吡咯烷-3,3'-吲哚酮类化合物.

\section{2 结果与讨论}

\section{1 反应条件的优化}

我们以对甲基苯胺衍生的 azaheptafulvene (1a)和 $N$-Boc-保护的 3-烯基吲哚酮(2a)作为反应的标准底物, 二氯甲烷作为反应溶剂. 当使用碱性氧化铝作为后处理 时柱层析介质时, 可以避免产物环庚三烯并吡咯烷3,3'-吲哚酮 (3a) 的异构化 ${ }^{[18]}$. 解决了产物纯化和异构化 问题后，我们以 $L$-脯氨酸和 2,6-二异丙基苯胺衍生的双 氮氧配体 L1 作为标准配体对不同中心金属进行考察. 以 L1-Zn $(\mathrm{OTf})_{2}$ 作为催化剂时, 能以 $99 \%$ 的收率、32： 68 的 $d r$ 值和 $34 \%$ 的 $e e$ 值获得相应的 [8+2]环加成产物 $3 \mathbf{a}$ (表 1 , Entry 1). 当以 $\mathrm{Sc}(\mathrm{OTf})_{3}$ 或 $\mathrm{La}(\mathrm{OTf})_{3}$ 作为金属前 体时, 产物的 $e e$ 值有所下降(表 1, Entries 2 3). 其它金 属前体如 $\mathrm{Mg}(\mathrm{OTf})_{2}, \mathrm{Co}\left(\mathrm{BF}_{4}\right)_{2} \bullet 6 \mathrm{H}_{2} \mathrm{O}$ 和 $\mathrm{Ni}\left(\mathrm{BF}_{4}\right)_{2} \bullet 6 \mathrm{H}_{2} \mathrm{O}$ 可 使反应的对映选择性得到极大的提高(表 1, Entries 4 6). 其中, $\mathrm{Ni}\left(\mathrm{BF}_{4}\right)_{2} \cdot 6 \mathrm{H}_{2} \mathrm{O}$ 能够给出高达 $95 \%$ 的 $e e$ 值和 几乎定量的产率, 但非对映选择性较低(表 1, Entry 6). 我们选择 $\mathrm{Ni}\left(\mathrm{BF}_{4}\right)_{2} \bullet 6 \mathrm{H}_{2} \mathrm{O}$ 作为中心金属, 进一步对手性 双氮氧配体的结构进行笁选, 以提高反应的 $d r$ 值.

我们从含二级胺的手性氨基酸(如 $L$-脯氨酸、 $L$-哌 啶-2 甲酸和 $L$-雷米普利中间体)和不同取代苯胺衍生物 合成了一系列手性双氮氧酰胺化合物. 固定双氮氧配体 中的氨基酸骨架为 $L$-脯氨酸骨架, 对酰胺部分的位阻效 应进行考察. 当双氮氧配体中酰胺部分苯环 2,6-位的取 代基位阻减小时, 即从异丙基变为乙基、甲基时, 产物 3a 的 $e e$ 值逐渐下降, 而非对映选择性虽没有很大提高, 
但主要异构体发生反转(表 1, Entries 6 9), 表明位阻效 应对调节催化剂的空间结构有很大影响. 因此固定 2,6二异丙基苯酰胺骨架, 对氨基酸骨架的影响进行考察. 结果显示: 当使用 $L$-雷米普利衍生的双氮氧配体 L6 时, 反应的 $d r$ 值可以达到 $80: 20$, 同时 $e e$ 值也略有所上升 (表 1, Entry 11). 因此, 我们选择 $L$-雷米普利衍生的双氮 氧配体 $\mathbf{L 6}$ 作为最佳配体. 以 $\mathbf{L 6}-\mathrm{Ni}\left(\mathrm{BF}_{4}\right)_{2} \cdot 6 \mathrm{H}_{2} \mathrm{O}$ 作为催 化剂, 可获得 99\%的收率、80:20 的 $d r$ 值和 97\%的 ee 值(表 1, Entry 11). 在此基础上降低催化剂用量至 1 $\mathrm{mol} \%$, 反应的 $d r$ 值和 $e e$ 值均有所上升(表 1, Entry 11). 继续降低催化剂用量到 $0.5 \mathrm{~mol} \%$, 反应的 $d r$ 值和 $e e$ 值 略有下降(表 1, Entry 12). 综上所述, 优化反应条件为: 1 $\mathrm{mol} \% \mathbf{L 6}-\mathrm{Ni}\left(\mathrm{BF}_{4}\right)_{2} \cdot 6 \mathrm{H}_{2} \mathrm{O}$ 作为催化剂, 二氯甲烷为溶剂, 反应温度 $35{ }^{\circ} \mathrm{C}$.

表 1 反应条件的优化

Table 1 Optimization of the reaction conditions

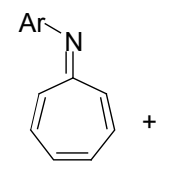

$\mathrm{Ar}=4-\mathrm{MeC}_{6} \mathrm{H}_{4}$

$1 \mathrm{a}$

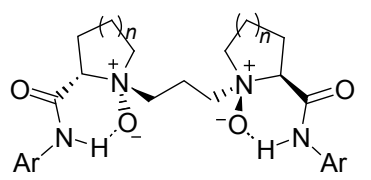

L1: $\mathrm{Ar}=2,6-i-\mathrm{Pr}_{2} \mathrm{C}_{6} \mathrm{H}_{3}, n=1$

L2: $\mathrm{Ar}=2,6-\mathrm{Et}_{2} \mathrm{C}_{6} \mathrm{H}_{3}, n=1$

L3: $\mathrm{Ar}=2,6-\mathrm{Me}_{2} \mathrm{C}_{6} \mathrm{H}_{3}, n=1$

L4: $\mathrm{Ar}=\mathrm{Ph}, n=1$

L5: $\mathrm{Ar}=2,6-i-\mathrm{Pr}_{2} \mathrm{C}_{6} \mathrm{H}_{3}, n=2$

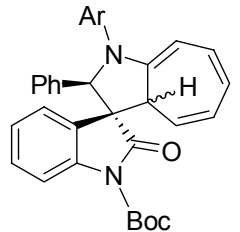

$3 a$

\begin{tabular}{ccccccc}
\hline Entry $^{a}$ & Metal & $\mathbf{L}$ & $x$ & $\mathrm{Yield}^{b} / \%$ & $d r^{c}$ & $e e^{c} / \%$ \\
\hline 1 & $\mathrm{Zn}(\mathrm{OTf})_{2}$ & $\mathbf{L 1}$ & 10 & 99 & $32: 68$ & 34 \\
2 & $\mathrm{Sc}(\mathrm{OTf})_{3}$ & $\mathbf{L 1}$ & 10 & 87 & $26: 74$ & 0 \\
3 & $\mathrm{La}(\mathrm{OTf})_{3}$ & $\mathbf{L 1}$ & 10 & 99 & $44: 56$ & 25 \\
4 & $\mathrm{Mg}\left(\mathrm{ClO}_{4}\right)_{2}$ & $\mathbf{L 1}$ & 10 & 99 & $28: 72$ & 91 \\
5 & $\mathrm{Co}\left(\mathrm{BF}_{4}\right)_{2} \bullet 6 \mathrm{H}_{2} \mathrm{O}$ & $\mathbf{L 1}$ & 10 & 99 & $44: 56$ & 88 \\
6 & $\mathrm{Ni}\left(\mathrm{BF}_{4}\right)_{2} \bullet 6 \mathrm{H}_{2} \mathrm{O}$ & $\mathbf{L 1}$ & 10 & 99 & $45: 55$ & 95 \\
7 & $\mathrm{Ni}\left(\mathrm{BF}_{4}\right)_{2} \bullet 6 \mathrm{H}_{2} \mathrm{O}$ & $\mathbf{L 2}$ & 10 & 96 & $58: 42$ & 91 \\
8 & $\mathrm{Ni}\left(\mathrm{BF}_{4}\right)_{2} \bullet 6 \mathrm{H}_{2} \mathrm{O}$ & $\mathbf{L 3}$ & 10 & 99 & $66: 34$ & 89 \\
9 & $\mathrm{Ni}\left(\mathrm{BF}_{4}\right)_{2} \bullet 6 \mathrm{H}_{2} \mathrm{O}$ & $\mathbf{L 4}$ & 10 & 99 & $63: 37$ & -30 \\
10 & $\mathrm{Ni}\left(\mathrm{BF}_{4}\right)_{2} \bullet 6 \mathrm{H}_{2} \mathrm{O}$ & $\mathbf{L 5}$ & 10 & 99 & $53: 47$ & 78 \\
11 & $\mathrm{Ni}\left(\mathrm{BF}_{4}\right)_{2} \bullet 6 \mathrm{H}_{2} \mathrm{O}$ & $\mathbf{L 6}$ & 10 & 99 & $80: 20$ & 97 \\
12 & $\mathrm{Ni}\left(\mathrm{BF}_{4}\right)_{2} \bullet 6 \mathrm{H}_{2} \mathrm{O}$ & $\mathbf{L 6}$ & 1 & 99 & $85: 15$ & 99 \\
13 & $\mathrm{Ni}\left(\mathrm{BF}_{4}\right)_{2} \bullet 6 \mathrm{H}_{2} \mathrm{O}$ & $\mathbf{L 6}$ & 0.5 & 99 & $83: 17$ & 94 \\
\hline
\end{tabular}

${ }^{a}$ Reaction conditions: metal $/ \mathbf{L}(1: 1), \mathbf{2 a}(0.05 \mathrm{mmol})$ and $\mathbf{1 a}(0.055 \mathrm{mmol})$ in $\mathrm{CH}_{2} \mathrm{Cl}_{2}(0.5 \mathrm{~mL})$ at $35{ }^{\circ} \mathrm{C}$ for $3 \mathrm{~h}$, and the product was purified by flash chromatography on basic $\mathrm{Al}_{2} \mathrm{O}_{3} .{ }^{b}$ Isolated yield. ${ }^{c}$ Determined by chiral HPLC.

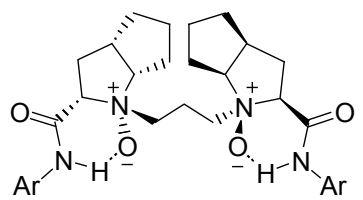

L6: $\mathrm{Ar}=2,6-i-\mathrm{Pr}_{2} \mathrm{C}_{6} \mathrm{H}_{3}$

\section{2 底物普适性考察}

在最佳反应条件下, 对 $[8+2]$ 环加成反应的底物普 适性进行考察, 结果见表 2 . 手性双酰胺一氮氧 L 6 - N i ( $\left(\mathrm{B} \mathrm{F}_{4}\right)_{2} \cdot 6 \mathrm{H}_{2} \mathrm{O}$ 催化体系对各种取代的 azaheptafulvenes 和 $N$-Boc-3-烯基吲哚酮的 [8+2]环加成 反应表现出优异的催化性能. 我们尝试了一系列的 azaheptafulvenes 底物, 包括供电基和吸电基取代的 $8 \pi$ 电子供体. 结果显示在 $1 \mathrm{~mol} \%$ 催化剂用量下, 能够以定 量的收率、较好的非对映选择性( $83: 17 \sim 86: 14 d r$ 值) 和优异的对映选择性( $97 \%$ \% 9 \% ee 值)获得相应的螺环 加成产物(表 2, Entries 1 4). 进一步对 $N$-Boc-3-烯基吲 哚酮烯芳基的取代基团进行考察. 结果显示: 催化体系 对烯芳基取代基的电子效应不敏感，无论吸电子还是供 电子取代基团，均能获得很好的收率、高的非对映选择 性和优异的对映选择性(表 2, Entries 5 10). 2-荟取代的 烯基吲哚酮也能够在此催化体系下获得 99\%收率，86：

表 2 底物普适性的考察

Table 2 Substrate scope of the reaction

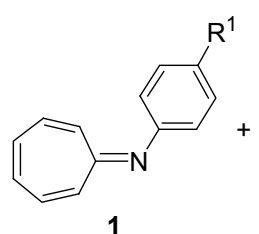<smiles>[R]C=C1C(=O)N(C(C)=O)c2ccc([R])cc21</smiles>

$\mathrm{L}-\mathrm{Ni}\left(\mathrm{BF}_{4}\right)_{2} \cdot 6 \mathrm{H}_{2} \mathrm{O}$ (1 $\mathrm{mol} \%)$

$\mathrm{CH}_{2} \mathrm{Cl}_{2}, 35^{\circ} \mathrm{C}$ $\left(\mathrm{Ar}=4-\mathrm{R}^{1} \mathrm{C}_{6} \mathrm{H}_{4}\right)$<smiles>[R]c1ccc2c(c1)[C@]1(C(=O)N2C(=O)OC(C)(C)C)C([R])C2C=CC=CC21</smiles>

3

\begin{tabular}{|c|c|c|c|c|c|c|c|}
\hline Entry $^{a}$ & $\mathrm{R}^{1}$ & $\mathrm{R}^{2}, \mathrm{R}^{3}$ & 3 & Time/h & Yield $^{b} / \%$ & $d r^{c}$ & $e e^{c} / \%$ \\
\hline 1 & $\mathrm{Me}$ & $\mathrm{Ph}, \mathrm{H}$ & $3 \mathbf{a}$ & 3 & 99 & $85: 15$ & 99 \\
\hline $2^{d}$ & $\mathrm{MeO}$ & $\mathrm{Ph}, \mathrm{H}$ & $3 \mathbf{b}$ & 3 & 99 & $83: 17$ & 97 \\
\hline 3 & $\mathrm{Cl}$ & $\mathrm{Ph}, \mathrm{H}$ & $3 \mathrm{c}$ & 3 & 99 & $86: 14$ & 98 \\
\hline 4 & $\mathrm{Br}$ & $\mathrm{Ph}, \mathrm{H}$ & 3d & 3 & 99 & $86: 14$ & 99 \\
\hline 5 & $\mathrm{Me}$ & $4-\mathrm{FC}_{6} \mathrm{H}_{4}, \mathrm{H}$ & $3 e$ & 3 & 96 & $87: 13$ & 99 \\
\hline 6 & $\mathrm{Me}$ & $3-\mathrm{ClC}_{6} \mathrm{H}_{4}, \mathrm{H}$ & $3 f$ & 3 & 97 & $86: 14$ & 99 \\
\hline 7 & $\mathrm{Me}$ & $4-\mathrm{ClC}_{6} \mathrm{H}_{4}, \mathrm{H}$ & $3 g$ & 3 & 99 & $87: 13$ & 98 \\
\hline 8 & $\mathrm{Me}$ & 4- $\mathrm{BrC}_{6} \mathrm{H}_{4}, \mathrm{H}$ & $3 \mathrm{~h}$ & 3 & 99 & $83: 17$ & 92 \\
\hline 9 & $\mathrm{Me}$ & $4-\mathrm{MeC}_{6} \mathrm{H}_{4}, \mathrm{H}$ & $3 \mathbf{i}$ & 3 & 92 & $87: 13$ & 99 \\
\hline 10 & $\mathrm{Me}$ & $3-\mathrm{MeOC}_{6} \mathrm{H}_{4}, \mathrm{H}$ & $3 \mathbf{j}$ & 3 & 99 & $84: 16$ & 99 \\
\hline 11 & $\mathrm{Me}$ & 2-Naph, H & $3 \mathbf{k}$ & 3 & 99 & $86: 14$ & 93 \\
\hline 13 & $\mathrm{Me}$ & $\mathrm{Ph}, \mathrm{F}$ & 31 & 3 & 99 & $79: 21$ & 99 \\
\hline 14 & $\mathrm{Me}$ & $\mathrm{Ph}, \mathrm{Br}$ & $3 \mathrm{~m}$ & 3 & 99 & $81: 19$ & 98 \\
\hline 15 & $\mathrm{Me}$ & $\mathrm{Ph}, \mathrm{MeO}$ & $3 \mathbf{n}$ & 3 & 99 & $81: 19$ & 99 \\
\hline
\end{tabular}

${ }^{a}$ Reaction conditions: $\mathrm{Ni}\left(\mathrm{BF}_{4}\right)_{2} \bullet 6 \mathrm{H}_{2} \mathrm{O} / \mathbf{L 6}(1: 1), 2(0.05 \mathrm{mmol})$ and $\mathbf{1}(0.055$ $\mathrm{mmol})$ in $\mathrm{CH}_{2} \mathrm{Cl}_{2}(0.5 \mathrm{~mL})$ for $3 \mathrm{~h}$, and 3 was purified by flash chromatography on basic $\mathrm{Al}_{2} \mathrm{O}_{3}$. The $d r$ and ee values were determined by chiral HPLC. ${ }^{b}$ Isolated yield. ${ }^{c}$ Determined by chiral HPLC. ${ }^{d}$ The $8 \pi$ component was generated in situ from the corresponding tetrafluoroborate salt with $\mathrm{Et}_{3} \mathrm{~N}$. 
$14 d r$ 值和 $93 \%$ ee 值(表 2, Entry 11). 吲哚酮环上 5 位取 代基的电子效应对收率和立体选择性没有显著影响，吸 电的和供电的取代基均能够很好的适合此催化体系(表 2, Entries 13 15).

值得一提的是, 当使用活性高的底物 20 作为 $2 \pi$ 电 子组分时, 反应可以获得高达 $97: 3$ 的 $d r$ 值和 99\%的 $e e$ 值(图 4a). 接下来, 为了考察此催化体系的可应用性, 我们在克级规模上合成螺环-吡咯烷-3,3'-吲哚酮 $\mathbf{3 c}$ (图 4b). 采用 2.0 毫摩尔的 $N$-Boc-3-烯基吲哚酮 $\mathbf{2 a}$ 和 1.02 摩尔当量的 1c, 在 $1 \mathrm{~mol} \%$ 的催化剂用量条件下反应 $4 \mathrm{~h}$, 以 $99 \%$ 的收率得到了 $1.07 \mathrm{~g}$ 的环加成产物 3c (99\%收率), 反应的对映和非对映选择性均能保持.

\section{3 结论}

我们通过 azaheptafulvenes 与 $N$-Boc-3-烯基吲哚䤊 的催化不对称 $[8+2]$ 环加成反应, 成功构建了系列环庚 三烯并吡咯烷-3,3'-吲哚酮类化合物. 在 $1 \mathrm{~mol} \%$ 手性双 氮氧酰胺 $\mathbf{L}-\mathrm{Ni}\left(\mathrm{BF}_{4}\right)_{2} \cdot 6 \mathrm{H}_{2} \mathrm{O}$ 配合物催化下, 能够以高收 率、较好的非对映选择性和优异的对映选择性得到目标 产物. 该反应条件温和, 无需氮气氛围, 可放大到克级 规模而不影响反应结果，具有好的应用前景.

\section{4 实验部分}

\section{1 催化剂溶液的配制}

在 $5.0 \mathrm{~mL}$ 容量瓶中称入 $\mathrm{Ni}\left(\mathrm{BF}_{4}\right)_{2} \bullet 6 \mathrm{H}_{2} \mathrm{O}(17.0 \mathrm{mg})$, L6 (35.0 mg), 加入无水 THF 定容至刻度, 得到澄清绿 色溶液。

\section{2 催化反应操作}

用微量注射器取 $50 \mu \mathrm{L}$ 上述催化剂溶液( $1 \mathrm{~mol} \%$ )加 入到磨口试管中, 真空除去 THF 溶液. 然后依次加入底 物 $N$-Boc-3-烯基吲哚酮 2a (0.05 mmol, $16.0 \mathrm{mg}$ ) 和 0.5 $\mathrm{mL}$ 二氯甲烷, 于 $35{ }^{\circ} \mathrm{C}$ 水浴下搅拌 $0.5 \mathrm{~h}$ 后, 加入底物 1a (1.05 equiv., $10.7 \mathrm{mg}$ ), 继续于 $35{ }^{\circ} \mathrm{C}$ 下搅拌, TLC 检 测反应进程. 反应结束后，反应液直接用碱性氧化铝进 行柱层析(氧化铝的上面和下面均铺少量硅藻土), 用石 油醚/二氯甲烷/乙酸乙酯混合溶剂 $(V: V: V=8: 8: 1)$ 进行洗脱, 得到浅黄色粘液(99\%收率). 手性 HPLC 测 定：DAICEL CHIRALCEL ID，正已烷/异丙醇 $=95 / 5$, 流速 $=1.0 \mathrm{~mL} / \mathrm{min}, \lambda=254 \mathrm{~nm}, t_{\mathrm{R}}: 5.897 \mathrm{~min}$ (minor), $6.700 \mathrm{~min}$ (major), $7.962 \mathrm{~min}$ (major), $8.422 \mathrm{~min}$ (minor).

\section{3 底物 azaheptafulvene 的合成}

将五氯化磷 $\left(\mathrm{PCl}_{5}, 20.82 \mathrm{~g}, 100 \mathrm{mmol}\right)$ 称入 $250 \mathrm{~mL}$ 圆底烧瓶中，倒入 $150 \mathrm{~mL}$ 二氯甲烷，放置于冰水浴中 冷却，产生大量的氯化氢，迅速将黄色的环庚三烯(液 体, $5.176 \mathrm{~mL}, 50 \mathrm{mmol}$ )滴入反应瓶中, 接尾气吸收装置 ( $\mathrm{NaOH}$ 水溶液), 剧烈搅拌反应 $3 \mathrm{~h}$ 后, 体系成浅黄色糊 状.

将 $100 \mathrm{~mL}$ 乙醇加入到 $250 \mathrm{~mL}$ 圆底烧瓶中, 并于冰 水浴中冷却; 将第一步中所得浅黄色糊状物用大口的布 氏漏斗于通风梪内抽滤，用二氯甲烷迅速洗涤，而后快 速将抽滤好的固体转移到已冷却的 $100 \mathrm{~mL}$ 乙醇中. 实 验现象：固体加入乙醇中，放热明显，并伴有刺刺声， 溶液颜色变深. 固体转移完毕, 将 $40 \%$ 的 $\mathrm{HBF}_{4}$ 溶液 (13.5 mL)迅速滴加到反应中去，反应数分钟内完毕，产 生大量白色固体. 白色固体经抽滤后, 用冷的乙醇洗涤, 抽干, 得到纯品为白色固体(6.2711 g). 此四氟硼酸盐性 质稳定. 所有经抽滤洗脱的酸性溶液用碱中和后妥善处 理.

将上述得到的四氟硼酸盐 $(6.2711 \mathrm{~g}, 35 \mathrm{mmol})$ 置于 $100 \mathrm{~mL}$ 的圆底烧瓶中，室温下加入 $10.0 \mathrm{~mL}$ 水，体系呈 白色糊状，未溶解. 将对甲基苯胺碾碎成粉末状，倒入 到反应瓶中，剧烈搅拌. 反应现象：白色随即消失，体 系呈褐色, 室温继续摚拌 $1 \mathrm{~h}$ 后, 体系变为黄红色. 将此 反应体系冷冻至 $-20{ }^{\circ} \mathrm{C}$, 停止搅拌, 半小时后, 烧瓶内 壁上析出黄色固体，烧瓶底部有少量黑色固体. 抽滤, (a)

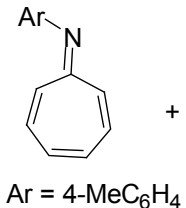

$1 \mathrm{a}$

(b)

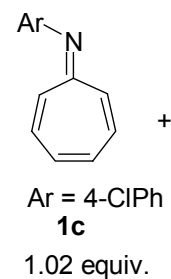

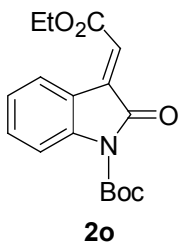

20

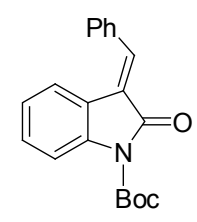

$2 a$

$2.0 \mathrm{mmol}$

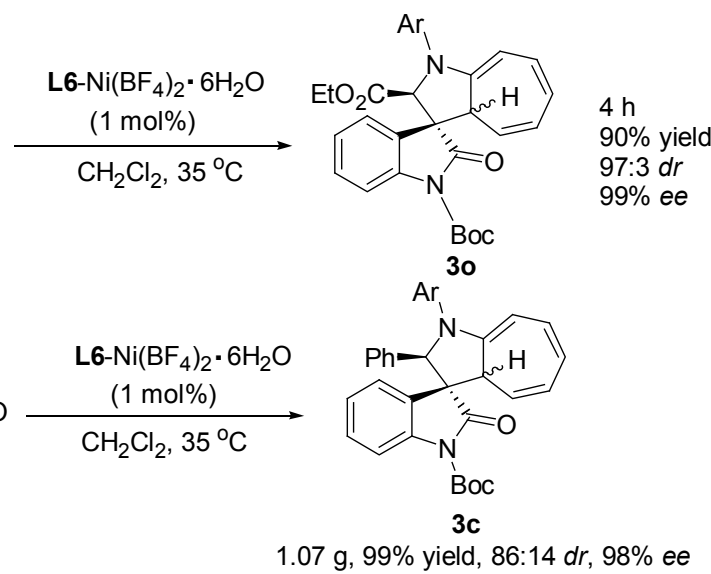

图 4 (a) 底物 20 的环加成; (b) 克级规模上合成环加成产物 $3 \mathrm{c}$

Figure 4 (a) The cycloaddition of $\mathbf{2 0}$; (b) the gram-scale synthesis of cycloadduct $\mathbf{3 c}$ 
用水洗涤, 得到黄色固体. 黄色固体用异丙醇进行重结 晶, 热溶冷析, 可得到针状橙色固体. 抽滤, 石油醚洗 涤, 真空干燥得橙色针状晶体(4.3358 g), 空气中稳定. 称上述橙色固体 $(4.3358 \mathrm{~g}, 15.31 \mathrm{mmol})$ 于 $100 \mathrm{~mL}$ 圆底烧瓶中, 加入 $23.0 \mathrm{~mL}$ 水和 $23.0 \mathrm{~mL}$ 二氯甲烷, 搅 拌并冷却于冰水浴中。随后缓慢滴加碳酸钠溶液 $(10 \%$ $\omega, 15.0 \mathrm{~mL})$, 并剧烈搅拌, 数分钟后, 反应完成. 有机 层变成橙色. 反应液用二氯甲烷萃取三次, 合并有机层, 无水硫酸镁干燥, 抽滤, 浓缩. 加石油醚、乙酸乙酯至浓 缩液, 析出部分黑色粘液. 将上层橙红色液体倾倒出来, 浓缩. 如此重复几次, 至再无黑色粘液析出. 橙红色浓 缩液用石油醚进行重结晶, 加热冷析(冷却至 $-20{ }^{\circ} \mathrm{C}$ ), 得橙红色固体, 抽滤, 石油醚洗涤, 抽干即可(1.85 g), 放置于 $-20{ }^{\circ} \mathrm{C}$ 冰箱中保存备用.

\section{References}

[1] (a) Bindra, J. S. The Alkaloids, Academic Press, New York, 1973, Vol. 14, p. 84; (b) Cui, C.-B.; Kakeya, H.; Osada, H. Tetrahedron 1996, 52, 12651; (c) Cui, C.-B.; Kakeya, H.; Osada, H. J. Antibiot. 1996, 49,832

[2] For reviews In Construction of 3,3'-Spirocyclic Oxindoles, see: (a) Marti, C.; Carreira, E. M. Eur. J. Org. Chem. 2003, 2209; (b) Galliford, C. V.; Scheidt, K. A. Angew. Chem., Int. Ed. 2007, 46, 8748; (c) Trost, B. M.; Brennan, M. K. Synthesis 2009, 3003; (d) Zhou, F.; Liu, Y.-L.; Zhou, J. Adv. Synth. Catal. 2010, 352, 1381; (e) Hong, L.; Wang, R. Adv. Synth. Catal. 2013, 355, 1023.

[3] For reviews on [3+2] cycloaddition of azomethine ylides, see: (a) Stanley, L. M.; Sibi, M. P. Chem. Rev. 2008, 108, 2887; (b) Adrio, J.; Carretero, J. C. Chem. Commun. 2011, 47, 6784; (c) Pellissier, H. Tetrahedron 2012, 68, 2197.

[4] For selected examples of synthesis chiral spiro[pyrrolidin3,3'-oxindoles] by using chiral substrates, see: (a) Sebahar, P. R.; Williams, R. M. J. Am. Chem. Soc. 2000, 122, 5666; (b) Sebahar, P. R.; Osada, H.; Usui, T.; Williams, R. M. Tetrahedron 2002, 58, 6311; (c) Onishi, T.; Sebahar, P. R.; Williams, R. M. Org. Lett. 2003, 5, 3135; (d) Lo, M. M.-C.; Neumann, C. S.; Nagayama, S.; Perlstein, E. O.; Schreiber, S. L. J. Am. Chem. Soc. 2004, 126, 16077.

[5] For other efforts on the asymmetric synthesis of spiro[pyrrolidin-3,3'-oxindoles], see: (a) Overman, L. E.; Rosen, M. D. Angew. Chem., Int. Ed. 2000, 39, 4596; (b) Trost, B. M.; Brennan, M. K. Org. Lett. 2006, 8, 2027.

[6] Chen, X.-H.; Wei, Q.; Luo, S.-W.; Xiao, H.; Gong, L.-Z. J. Am. Chem. Soc. 2009, 131, 13819.

[7] (a) Antonchick, A. P.; Gerding-Reimers, C.; Catarinella, M.; Schürmann, M.; Preut, H.; Ziegler, S.; Rauh, D.; Waldmann, H. Nat. Chem. 2010, 2, 735; (b) Antonchick, A. P.; Schuster, H.; Bruss, H.; Schürmann, M.; Preut, H.; Rauh, D.; Waldmann, H. Tetrahedron 2011, 67, 10195; (c) Liu, T.-L.; Xue, Z.-Y.; Tao, H.-Y.; Wang, C.-J. Org. Biomol. Chem. 2011, 9, 1980; (d) Awata, A.; Arai, T. Chem.-Eur. J. 2012, 18, 8278; (e) Wang, L.; Shi, X.-M.; Dong, W.-P.; Zhu, L.-P.; Wang, R. Chem. Commun. 2013, 49, 3458.

[8] For selected examples on catalytic asymmetric synthesis of other spiro-oxindoles, see: (a) Trost, B. M.; Cramer, N.; Silverman, S. M. J. Am. Chem. Soc. 2007, 129, 12396; (b) Bui, T.; Syed, S.; Barbas III, C. F. J. Am. Chem. Soc. 2009, 131, 8758; (c) Galzerano, P.; Bencivenni, G.; Pesciaioli, F.; Mazzanti, A.; Giannichi, B.; Sambri, L.; Bartoli, G.; Melchiorre, P. Chem.-Eur. J. 2009, 15, 7846; (d) Jiang, X. X.; Cao, Y. M.; Wang, Y. Q.; Liu, L. P.; Shen, F. F.; Wang, R. J. Am. Chem. Soc. 2010, 132, 15328; (e) Wang, L.-L.; Peng, L.; Bai, J.-F.; Huang, Q.-C.; Xu, X.-Y.; Wang, L.-X. Chem. Commun. 2010, 46, 8064; (f) Chen, W.-B.; Wu, Z.-J.; Hu, J.; Cun, L.-F.; Zhang, X.-M.; Yuan, W.-C. Org. Lett. 2011, 13, 2472; (g) Liu, Y.-K.; Nappi, M.; Arceo, E.; Vera, S.; Melchiorre, P. J. Am. Chem. Soc. 2011, 133, 15212; (h) Shen, L.-T.; Shao, P.-L.; Ye, S. Adv. Synth. Catal. 2011, 353, 1943; (i) Peng, J.; Huang, X.; Jiang,
L.; Cui, H.-L.; Chen, Y.-C.; Org. Lett. 2011, 13, 4584; (j) Li, Y.-M.; Li, X.; Peng, F.-Z.; Li, Z.-Q.; Wu, S.-T.; Sun, Z.-W.; Zhang, H.-B.; Shao, Z.-H. Org. Lett. 2011, 13, 6200.

[9] For reviews of higher-order cycloadditions, see: (a) Rigby, J. H. Acc. Chem. Res. 1993, 26, 579; (b) Harmata, M. Acc. Chem. Res. 2001, 34, 595; (c) Harmata, M. Adv. Synth. Catal. 2006, 348, 2297; (d) Nair, V.; Abhilash, K. G. Top. Heterocycl. Chem. 2008, 13, 173; (e) Nair, V.; Abhilash, K. G. Synlett 2008, 301; (f) Lohse, A. G.; Hsung, R. P. Chem.-Eur. J. 2011, 17, 3812; (g) Pellissier, H. Adv. Synth. Catal. 2011, 353, 189; (h) Ylijoki, K. E. O.; Stryker, J. M. Chem. Rev. 2013, 113, 2244.

[10] For an example of a catalytic asymmetric [6+2] cycloaddition with fulvenes as $6 \pi$ components, see: (a) Hayashi, Y.; Gotoh, H.; Honma, M.; Sankar, K.; Kumar, I.; Ishikawa, H.; Konno, K.; Yui, H.; Tsuzuki, S.; Uchimaru, T. J. Am. Chem. Soc. 2011, 133, 20175; For examples of catalytic asymmetric [6+3] cycloadditions with fulvenes as $6 \pi$ components, see: (b) Potowski, M.; Bauer, J. O.; Strohmann, C.; Antonchick, A. P.; Waldmann, H. Angew. Chem., Int. Ed. 2012, 51, 9512; (c) He, Z.-L.; Teng, H.-L.; Wang, C.-J. Angew. Chem., Int. Ed. 2013, 52, 2934.

[11] For examples of catalytic asymmetric [5+2] cycloadditions, see: (a) Wender, P. A.; Haustedt, L. O.; Lim, J.; Love, J. A.; Williams, T. J.; Yoon, J.-Y. J. Am. Chem. Soc, 2006, 128, 6302; (b) Shintani, R.; Nakatsu, H.; Takatsu, K.; Hayashi, T. Chem.-Eur. J. 2009, 15, 8692.

[12] For examples of catalytic asymmetric [4+3] cycloadditions, see: (a) Harmata, M.; Ghosh, S. K.; Hong, X.; Wacharasindhu, S.; Kirchhoefer, P. J. Am. Chem. Soc. 2003, 125, 2058; (b) Huang, J.; Hsung, R. P. J. Am. Chem. Soc. 2005, 127, 50; (c) Dai, X.; Davies, H. M. L. Adv. Synth. Catal. 2006, 348, 2449; (d) Reddy, R. P.; Davies, H. M. L. J. Am. Chem. Soc. 2007, 129, 10312; (e) Gulías, M.; Durán, J.; López, F.; Castedo, L.; Mascareñas, J. L. J. Am. Chem. Soc. 2007, 129, 11026; (f) Shintani, R.; Murakami, M.; Hayashi, T. J. Am. Chem. Soc. 2007, 129, 12356; (g) Schwartz, B. D.; Denton, J. R.; Lian, Y.; Davies, H. M. L.; Williams, C. M. J. Am. Chem. Soc. 2009, 131, 8329; (h) Shintani, R.; Murakami, M.; Tsuji, T.; Tanno, H.; Hayashi, T. Org. Lett. 2009, 11, 5642; (i) Alonso, I.; Faustino, H.; López, F.; Mascareñas, J. L. Angew. Chem., Int. Ed. 2011, 50, 11496.

[13] For examples of [8+2] cycloadditions with dienylisobenzofurans as $8 \pi$ components, see: (a) Luo, Y.; Herndon, J. W.; Cervantes-Lee, F. J. Am. Chem. Soc. 2003, 125, 12720; (b) Zhang, L.; Wang, Y.; Buckingham, C.; Herndon, J. W. Org. Lett. 2005, 7, 1665; (c) Chen, Y.; Ye, S.; Jiao, L.; Liang, Y.; Sinha-Mahapatra, D. K.; Herndon, J. W.; Yu, Z.-X. J. Am. Chem. Soc. 2007, 129, 10773; (d) Roy, P.; Ghorai, B. K. Tetrahedron Lett. 2011, 52, 5668.

[14] For examples of [8+2] cycloadditions with indolizines as $8 \pi$ components, see: (a) Godfrey, J. C. J. Org. Chem. 1959, 24, 581; (b) Galbraith, A.; Small, T.; Barnes, R. A.; Boekelheide, V. J. Am. Chem. Soc. 1961, 83, 453; (c) Aginagalde, M.; Vara, Y.; Arrieta, A.; Zangi, R.; Cebolla, V. L.; Delgado-Camón, A.; Cossío, F. P. J. Org. Chem. 2010, 75, 2776.

[15] For examples of [8+2] cycloadditions with benzothietes as $8 \pi$ components, see: (a) Kanakarajan, K.; Meier, H. J. Org. Chem. 1983, 48, 881; (b) Meier, H.; Mayer, A. Angew. Chem., Int. Ed. Engl. 1994, 33, 465; (c) Meier, H.; Rose, B.; Schollmeyer, D. Liebigs Ann. 1997, 1173.

[16] For examples of [8+2] cycloadditions with heptafulvenes as $8 \pi$ components, see: (a) Doering, W. von E.; Wiley, D. W. Tetrahedron 1960, 11, 183; (b) Liu, C.-Y.; Mareda, J.; Houk, K. N.; Fronczek, F. R. J. Am. Chem. Soc. 1983, 105, 6714; (c) Liu, C.-Y.; Shie, H.-Y.; Chen, S.-Y.; You, C.-Y.; Wang, W.-C.; Hua, L.-N.; Yang, H.-J.; Tseng, C.-M. Tetrahedron 1997, 53, 17275.

[17] For an example of a catalytic asymmetric [4+2] cycloaddition with tropones as $4 \pi$ components, see: (a) Li, P.; Yamamoto, H. J. Am. Chem. Soc. 2009, 131, 16628; For examples of catalytic asymmetric $[6+3]$ cycloadditions with tropones as $6 \pi$ components, see: $(b)$ Trost, B. M.; McDougall, P. J.; Hartmann, O.; Wathen, P. T. J. Am. Chem. Soc. 2008, 130, 14960; (c) Trost, B. M.; McDougall, P. J. Org. Lett. 2009, 11, 3782; For example of a catalytic asymmetric [6 $+4]$ cycloaddition with tropones as $6 \pi$ components, see: (d) Rigby, J. H.; Fleming, M. Tetrahedron Lett. 2002, 43, 8643 .

[18] Xie, M. S.; Liu, X. H.; Wu, X. X.; Cai, Y. F.; Lin, L. L.; Feng, X. M. Angew. Chem., Int. Ed. Engl. 2013, 52, 5604.

[19] For examples of tropone as the $8 \pi$ component, see: (a) Kumar, K.; Kapur, A.; Ishar, M. P. S. Org. Lett. 2000, 2, 787; (b) Okamoto, J.; 
Yamabe, S.; Minato, T.; Hasegawa, T.; Machiguchi, T. Helv. Chim. Acta 2005, 88, 1519; (c) Nair, V.; Poonoth, M.; Vellalath, S.; Suresh, E.; Thirumalai, R. J. Org. Chem. 2006, 71, 8964.

[20] For examples of tropothione as the $8 \pi$ component, see: (a) Machiguchi, T.; Hasegawa, T.; Otani, H.; Ishii, Y. J. Chem. Soc., Chem. Commun. 1987, 1375; (b) Machiguchi, T. Tetrahedron 1995, 51, 1133; (c) Nair, V.; Abhilash, K. G.; Suresh, E. Tetrahedron Lett. 2006, 47, 9329; (d) Rivero, A. R.; Fernández, I.; Sierra, M. A. J. Org. Chem. 2012, 77, 6648.

[21] For examples of azaheptafulvenes as $8 \pi$ components see: (a) Yamamoto, K.; Kajigaeshi, S.; Kanemasa, S. Chem. Lett. 1977, 85; (b) Yamamoto, K.; Kajigaeshi, S.; Kanemasa, S. Chem. Lett. 1977, 91;

(c) Truce, W. E.; Shepherd, J. P. J. Am. Chem. Soc. 1977, 99, 6453; (d) Nair, V.; Abhilash, K. G. Tetrahedron Lett. 2006, 47, 8707; (e) Lage, M. L.; Fernández, I.; Sierra, M. A.; Torres, M. R. Org. Lett. 2011, 13, 2892.
[22] (a) Sanechika, K.; Kajigaeshi, S.; Kanemasa, S. Chem. Lett. 1977, 861; (b) Ishizu, T.; Harano, K.; Yasuda, M.; Kanematsu, K. J. Org. Chem. 1981, 46, 3630; (c) Hayakawa, K.; Nishiyama, H.; Kanematsu, K. J. Org. Chem. 1985, 50, 512; (d) Barluenga, J. García-Rodríguez, J.; Martínez, S.; Suárez-Sobrino, Á. L.; Tomás, M. Chem.-Asian J. 2008, 3, 767; (e) Barluenga, J.; García-Rodríguez, J.; Suárez-Sobrino, Á. L.; Tomás, M. Chem.-Eur. J. 2009, 15, 8800

[23] For selected examples using chiral $N, N^{\prime}$-dioxide ligands, see: (a) Liu, X. H.; Lin, L. L.; Feng, X. M. Acc. Chem. Res. 2011, 44, 574; (b) Hassner, A.; Namboothiri, I. In Organic Syntheses Based on Name Reactions, 3rd ed., Elsevier, Oxford, 2011, 408; (c) Zheng, K.; Lin, L. L.; Feng, X. M. Acta Chim. Sinica 2012, 70, 1785. (郑柯, 林丽丽, 冯小明, 化学学报, 2012, 70, 1785.) (d) Zhao, J. N.; Liu, X. H.; Luo, W. W.; Xie, M. S.; Lin, L. L.; Feng, X. M. Angew. Chem., Int. Ed. Engl. 2013, 52, 3473. 